\title{
Collaboration of Internal Medicine Physicians with Patients and Other Health Care Providers in the Diagnostic Process
}

\author{
Thilan P. Wijesekera, MD, Lisa Sanders, MD, and Donna M. Windish, MD, MPH
}

J Gen Intern Med 34(7):1083-5

DOI: $10.1007 / \mathrm{s} 11606-019-04865-3$

(c) Society of General Internal Medicine 2019

\section{INTRODUCTION}

Given the increasing complexity of patients, the multitude of choices for diagnostic testing, and the specialization of providers, team-based care is an increasingly important part of the diagnostic process. 1,2 "Effective teamwork" is a core goal in the National Academy of Medicine's (NAMs) report "Improving Diagnosis in Health Care," to reduce diagnostic errors. ${ }^{3}$ Our study evaluated how internal medicine physicians are partnering with four underutilized members of the diagnostic team highlighted in the NAM report: patients, interdisciplinary teams, radiologists, and pathologists.

\section{METHODS}

\section{Setting and Participants}

Between June 2016 and March 2017, we surveyed internal medicine residents and attendings across nine residency training programs at six Connecticut hospitals. Questions assessed the frequency of collaboration with patients, interdisciplinary teams, radiologists, and pathologists with response choices: every patient, $\geq 2$ times per day, once daily, $\geq 2$ times per week, once weekly, once per month, once every few months, and never.

\section{Survey Administration}

We identified 484 potential participants who received anonymous paper-based surveys or electronic surveys via e-mail. We used summary statistics and Pearson's $\chi$ test for analyses. The Yale-New Haven Hospital Institutional Review Board approved the study.

$\overline{\text { Prior Presentations Oral Presentation: Diagnostic Error in Medicine 10th }}$ International Conference (10/8/2017)

Poster Presentation: Society of General Internal Medicine, New England Regional Meeting (3/9/2018)

Published online March 7, 2019

\section{RESULTS}

Fifty-five percent (266/484) of physicians completed the survey. The response rate was $49.4 \%$ (196/397) for trainees and $80.5 \%(70 / 87)$ for attendings. Most respondents were trainees $(73.7 \%, 196 / 266)$ and from university-affiliated hospitals $(71.4 \%, 190 / 266)$. Due to the low responses of some questions, answer choices were consolidated into four categories (Figs. 1 and 2).

Physicians infrequently communicated about the diagnostic process with their patients (Fig. 1). Fewer than half $(45.4 \%, 118 / 260)$ reported explaining their diagnostic thinking process with every patient (Fig. 1). Even fewer $(27.9 \%, 70 / 251)$ discussed a diagnostic error with every patient when they occurred (Fig. 1). Barely one in ten $(13.7 \%, 36 / 262)$ always performed "teach back," where they asked every patient to explain their plan of care back to them to gauge patients' level of understanding (Fig. 1).

Physicians inconsistently worked with other health care providers (Fig. 2). While most reported daily collaboration with interdisciplinary teams in the inpatient setting $(83.9 \%, 214 / 255)$, fewer worked with interdisciplinary teams in the outpatient setting $(50.4 \%, 118 / 234)$. When collaborating with subspecialists, $70.5 \%(182 / 258)$ of respondents in the inpatient setting reported at least weekly communication with radiologists, compared to $13.2 \%(31 / 235)$ in the outpatient setting (Fig. 2). Respondents were even less likely to communicate with pathologists, with $17.5 \%$ (45/257) reporting never having discussed results with a pathologist for in-hospital patients and $52.1 \%(122 / 234)$ never collaborating on patients outside the hospital (Fig. 2).

Differences in responses occurred based on years of experience and gender. Residents were less likely than attendings to collaborate with radiologists in the outpatient setting at least weekly $(8.1 \%$ vs. $32.0 \% ; p<0.001)$ and to ever collaborate with pathologists in the outpatient setting $(41.8 \%$ vs. $70.0 \% ; p<0.001)$. Female physicians were more likely to explain their diagnostic process with every patient $(56.0 \%$ vs. $40.8 \%$; $p=$ $0.055)$ and to use teach back in every patient encounter $(25.0 \%$ vs. $7.7 \%$; $p=0.003)$. There were no other differences in subgroup analyses. 


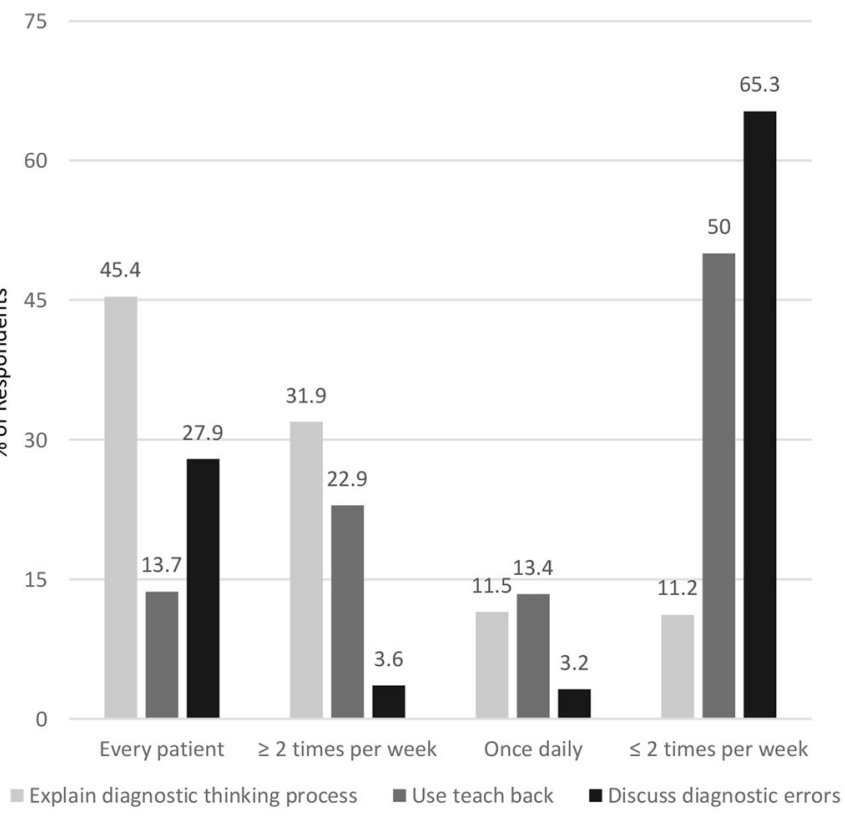

NOTE: Teach back was defined as "ask patients to explain plan of care back to you"

Figure 1 Cumulative responses of all physicians when asked how often they explain their diagnostic process, use teach back, and discuss diagnostic errors with their patients.

\section{DISCUSSION}

In our study of internal medicine physicians assessing their collaboration during the diagnostic process, we found that physicians did not communicate consistently with interdisciplinary teams, subspecialists, or patients.

The composition of the diagnostic team is changing. ${ }^{2}$ For that reason, it is concerning that in the outpatient setting, interdisciplinary communication was not regularly occurring. Similarly troubling, physicians were not directly communicating with radiologists and pathologists, especially junior physicians, potentially missing an opportunity for more nuanced descriptions of testing and results. ${ }^{4,5}$

The most alarming finding was the lack of assessing patient understanding or communicating with patients about diagnostic errors. Patient engagement barriers already include access, language, and literacy. ${ }^{3}$ Failure of physicians to explain their diagnostic thinking, ensure understanding through teach back, and discuss diagnostic errors risks further exacerbating those limitations. Interestingly, female physicians in our study reported engaging more with patients in the diagnostic process.

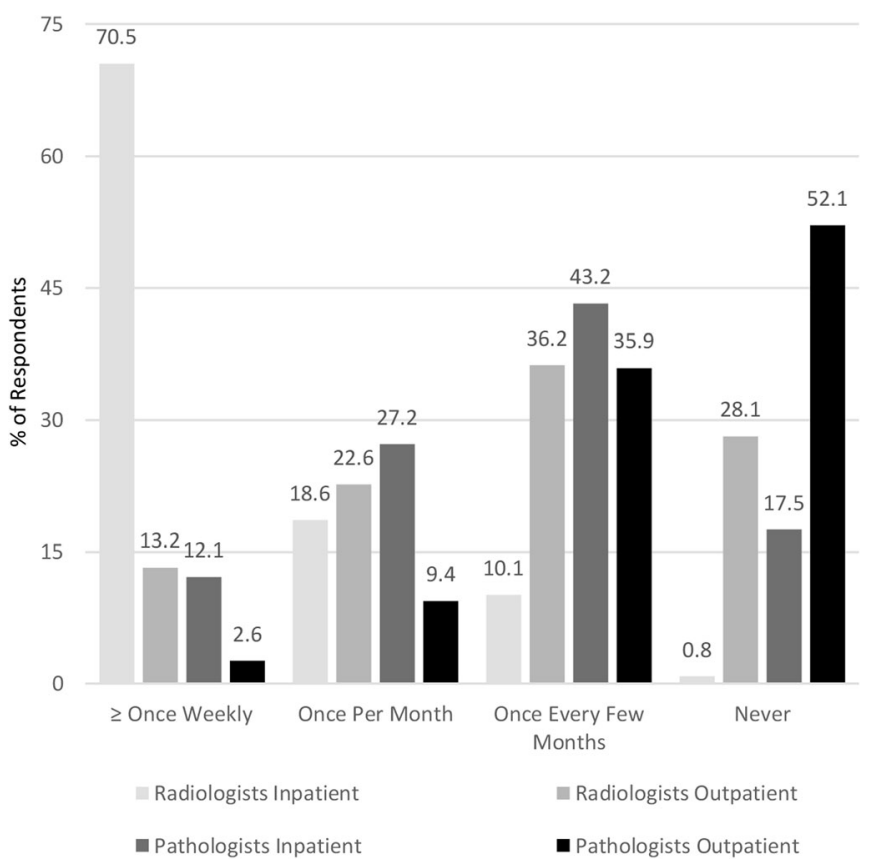

Figure 2 Cumulative responses of all physicians when asked how often they collaborate with radiologists or pathologists. 
This finding may need further exploration in light of a recent study showing that inpatients with female physicians had lower mortality and readmissions. ${ }^{6}$

An important study limitation was the likelihood of recall bias and the lack of patient perception about diagnostic communication. Moving forward, physicians should aim to include all participants involved in the diagnostic process to reduce diagnostic errors and potential patient harm.

Corresponding Author: Thilan P. Wijesekera, MD; Yale University School of Medicine, Harkness Hall A, Rm 306A, 367 Cedar Street, New Haven, CT 06510, USA (e-mail: thilan.wijesekera@yale.edu).

\section{Compliance with Ethical Standards:}

The Yale-New Haven Hospital Institutional Review Board approved the study.

Conflict of Interest: The authors declare that they do not have a conflict of interest.
Publisher's Note: Springer Nature remains neutral with regard to jurisdictional claims in published maps and institutional affiliations.

\section{REFERENCES}

1. Schiff GD. Diagnosis and diagnostic errors: time for a new paradigm. BMJ Gual Saf 2013; 23:1-3.

2. Graber ML, Rusz D, Jones ML, et al. The new diagnostic team. Diagnosis 2017; 4:225-238.

3. National Academies of Sciences, Engineering, and Medicine. Improving diagnosis in health care. Washington, DC: The National Academies Press, 2015.

4. Dickerson EC, Alam HB, Brown RK, et al. Collaborative MRQ. In-person communication between radiologists and acute care surgeons leads to significant alterations in surgical decision making. J Am Coll Radiol 2016; 13:943-949.

5. Powsner SM, Costa J, Homer RJ. Clinicians are from Mars and pathologists are from Venus: clinician interpretation of pathology reports. Arch Pathol Lab Med 2000; 124:1040-1046.

6. Tsugawa Y, Jena AB, Figueroa JF, Orav EJ, Blumenthal DM, Jha AK. Comparison of Hospital Mortality and Readmission Rates for Medicare Patients Treated by Male vs Female Physicians. JAMA Intern Med 2017; 177:206-13. 